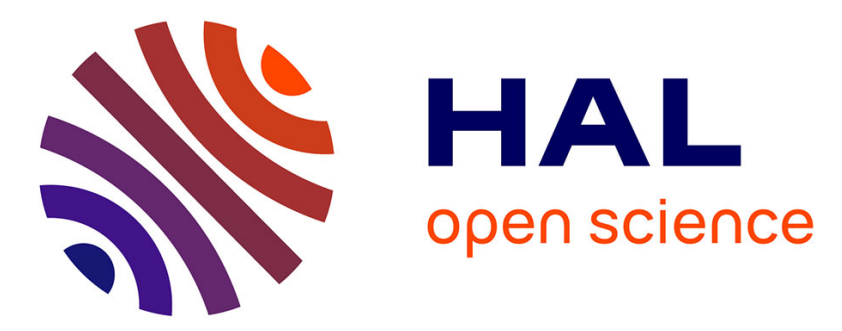

\title{
Frequency warping for waveguide characterization with a single hydrophone
}

Julien Bonnel, Barbara Nicolas, Jerome I. Mars, Dominique Fattaccioli

\section{To cite this version:}

Julien Bonnel, Barbara Nicolas, Jerome I. Mars, Dominique Fattaccioli. Frequency warping for waveguide characterization with a single hydrophone. OCEANS 2009 - OCEANS '09 MTS/IEEE. Marine Technology for our Future: Global and Local Challenges, Oct 2009, Biloxi, United States. pp.n/c. hal-00440519

\section{HAL Id: hal-00440519 https://hal.science/hal-00440519}

Submitted on 11 Dec 2009

HAL is a multi-disciplinary open access archive for the deposit and dissemination of scientific research documents, whether they are published or not. The documents may come from teaching and research institutions in France or abroad, or from public or private research centers.
L'archive ouverte pluridisciplinaire HAL, est destinée au dépôt et à la diffusion de documents scientifiques de niveau recherche, publiés ou non, émanant des établissements d'enseignement et de recherche français ou étrangers, des laboratoires publics ou privés. 


\section{Frequency warping for waveguide characterization with a single hydrophone}

\author{
Julien Bonnel \\ GIPSA-Lab / DIS \\ Grenoble INP, France \\ Email: julien.bonnel@gipsa-lab.inpg.fr
}

\author{
Barbara Nicolas \\ Jérôme I. Mars \\ GIPSA-Lab / DIS
}

Grenoble INP, France

\author{
Dominique Fattaccioli \\ Centre Technique des Systèmes Naval \\ DGA (Toulon), France
}

\begin{abstract}
This paper presents a new signal processing tool: frequency warping, and its application in waveguide characterization. It can be applied in the context of signals recorded in shallow water $(0-400 \mathrm{~m})$ for an impulsive low-frequency source $(0-200 \mathrm{~Hz})$ and a single static receiver. In this configuration, propagation is described by modal theory: the recorded pressure field can be decomposed into several modes. Modes are non linear-frequency modulations which share a common frequency band. When the radial distance between source and receiver is smaller than $15 \mathrm{~km}$, modes are also overlapped in time on the receiver. In this case, the recorded signal cannot be represented using classical time frequency representations and adaptive signal processing is required. Frequency warping processing transforms a give mode into a Dirac in time, using a priori information of the environment. As it is sensitive to environment mismatch, it can also be used to perform waveguide characterization. First, modal propagation is quickly reviewed. Secondly, it is shown that environment information is embedded in the timefrequency structure of the modes, but that adaptive signal processing is required to access it. Then, frequency warping processing is presented, both theoretically and experimentally. Finally, it is shown that frequency warping can be used to perform environment characterization.
\end{abstract}

\section{INTRODUCTION}

After decades of study about it, underwater acoustics is still a very topical issue. It is classically used to perform inversion of the environment or source localization using at least an array of hydrophones as receivers. Arrays utilization increases the signal to noise ratio and brings more information than single receiver configuration thanks to distance aperture. However, it is quite expensive and time consuming to deploy an array during a sea experiment. This is why now several studies have been done using only a sparse network of hydrophones [1] [2] [3]. Other studies have been done using a single fixed receiver. This configuration is the most simple for operational context, but it reduces drastically the signal to noise ratio and the quantity of available information. To overcome this, broadband sources are used to take most benefit of frequency diversity [4] [5]. This paper presents a new signal processing method (frequency warping) for this configuration (a single static broadband source and a single static receiver) and its application for waveguide characterization. Moreover, the context of this study narrows to impulsive low frequency source (0-200 Hz) and shallow water environment $(0-400 \mathrm{~m})$.

Warping is a suitable signal processing tool for underwater signal studies. Time warping processing as been presented and used in [2] and [6]. Frequency warping is a new processing which transforms a given mode into a Dirac (in time domain). It has first been developed in [7]. However, time and frequency warpings presented in the literature are based on isovelocity model for the environment. This paper presents a new frequency warping processing which can use any waveguide as a priori information on the environment.

First, modal propagation is quickly reviewed to cover necessary notions. Secondly, time-frequency tools are presented and it is shown that they are not sufficient to adequately represent an underwater signal. Then, frequency warping processing is presented in details, both theoretically and experimentally. Finally, first applications of frequency warping as a new tool to perform waveguide characterization are presented.

\section{MODAL PROPAGATION}

As stated in the introduction, normal mode theory is the most suitable propagation model for shallow water $(0-400 \mathrm{~m})$ and low frequencies $(0-200 \mathrm{~Hz})$. It is quickly reviewed in this section to introduce notions and equations useful for the rest of the paper.

In a range independent environment, for an impulsive source at depth $z_{s}$ and a receiver at depth $z_{r}$ separated by a radial distance $r$, the spectrum $Y(f)$ of the received signal $y(t)$ is [8] :

$$
Y(f) \approx Q \sum_{m=1}^{N} \Psi_{m}\left(f, z_{s}\right) \Psi_{m}\left(f, z_{r}\right) \frac{e^{j k_{r m}(f) r}}{\sqrt{k_{r m}(f) r}}
$$

where $N$ is the number of modes, $\Psi_{m}$ is the modal function of mode $m$ (which is a real function depending on frequency $f$ and on depth $z$, but only values for $z_{s}$ and $z_{r}$ are taken into account), $k_{r m}(f)$ the radial wavenumber of mode $m$ (which is supposed to be real as the evanescent modes are not taken in account), and $Q=\frac{e^{j \pi / 4}}{\sqrt{8 \pi} \rho\left(z_{s}\right)}$ (with $\rho\left(z_{s}\right)$ the water density at the source depth). The influence of the environment is notably embedded into the wavenumbers $k_{r m}$.

Each component of the sum in equation 1 represents the contribution of a given mode. For a given mode $m$ (i.e. a component in equation 1), group speed $v_{g m}$ is defined by:

$$
v_{g m}(f)=2 \pi \frac{\partial f}{\partial k_{r m}}
$$


For mode number $m, v_{g m}(f)$ describes the propagation speed of frequency $f$. Group speed is a useful notion which will be used in section III to study the time-frequency representation of the received signal.

\section{TIME-FREQUENCY TOOLS}

In the case of a range independent environment, a theoretical and sparse time-frequency structure of the received signal can be defined by considering that frequency $f$ of a given mode $m$ travels at speed $v_{g m}(f)$ in between a source at depth $z_{s}$ and a receiver at depth $z_{r}$ separated by a radial distance $r$ :

$$
T F S(t, f)=\sum_{m=1}^{N} \alpha\left(m, f, r, z_{s}, z_{r}\right) \delta\left(t-\frac{r}{v_{g m}(f)}\right)
$$

where $\delta(t)$ is the Dirac distribution describing the localization of the time-frequencies structures and $\alpha$ is the attenuation term describing their amplitude. An example of the TFS of the received signal recorded in a Pekeris waveguide is presented in Figure 1. In this TFS, each curve represents a mode, i.e. a component of the sum in equation 1 . Note that the extreme lower frequency part of each mode (between the cutoff frequency and the Airy phase [8]) is not represented as there is almost no energy in this frequency band. It is noticeable that modes are non-stationary and share a common band of frequencies. Moreover if the distance $r$ is smaller than $20 \mathrm{~km}$ modes, several modes exist for a given recording time (as it is the case on Figure 1). Consequently, it is reasonable to study recorded signal in time-frequency plan.

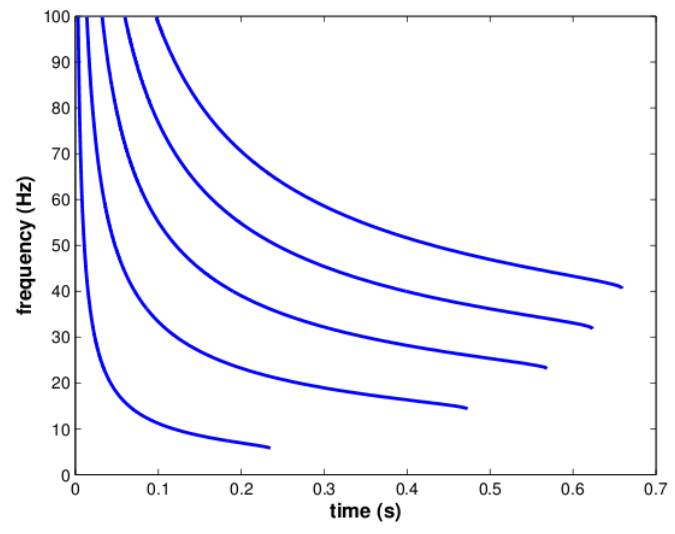

Fig. 1. Theoretical time-frequency representation of the received signal in a Pekeris waveguide

The curved structure of the modes is defined by the group velocities as shown by equation 3 and group velocities are linked to wavenumbers by equation 2 . As wavenumbers carry information about environment, the TFS also carry information about environment. However, the mode curves are close from each other. Because of that, classical time-frequency representations are not adapted to represent received signal. Indeed, interferences between structures would mix with the signal for
Wigner-Ville class representations and time-frequency uncertainty would mix modes for spectrogram class representations. An example of spectrogram for a simulated signal is presented in Figure 2. The simulated environment is a noisy Pekeris waveguide (presented in details in section V-B) with a signal to noise ratio of $5 \mathrm{~dB}$. One can guess the TFS on the spectrogram, but it is not clear. Adaptive signal processing is required.

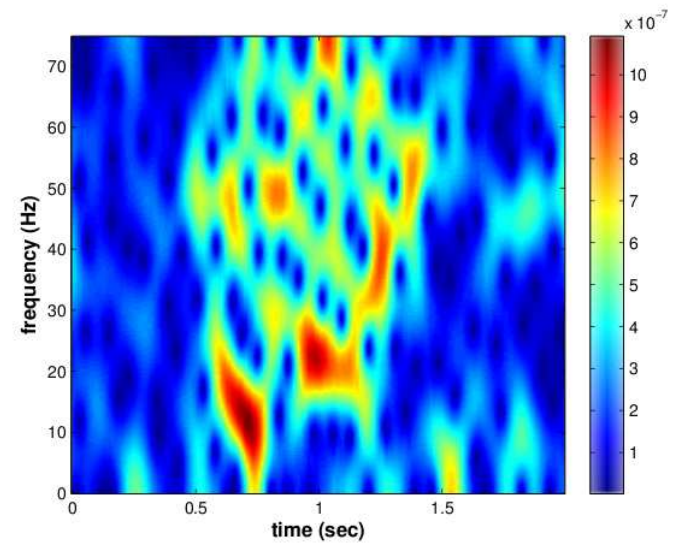

Fig. 2. Spectrogram of the simulated signal in a Pekeris waveguide $(\mathrm{SNR}=5 \mathrm{~dB})$

\section{FREQUENCY WARPING}

In [9], an adapted time-frequency representation matching the dispersion is presented. In this study, the received signal is transformed using warping processing so that it can be represented using classical time-frequency representations. Frequency warping is a new processing which transforms a given mode into a Dirac (in time domain).

\section{A. Mathematical aspects}

Mathematically, frequency warping processing is mainly a substitution. Let consider a function $g$ of the variable $x$. The warped version $\mathbf{W}_{w} g$ of $g$ is given by:

$$
\mathbf{W}_{w} g(x)=\sqrt{\left|\frac{d w(x)}{d x}\right|} g[w(x)]
$$

with $w$ the warping function. The variable $x$ is substituted by $h(x)$, and $g[w(x)]$ is multiplied by the square root term so that $\mathbf{W}_{h} g$ and $g$ have the same energy. This allows a warping interpretation in term of unitary equivalence [10]. This will not be reviewed in this article as it does not bring any physical interpretation about the warping. In our case, $g$ is the spectrum of the received signal, and $x$ is frequency $f$. Consequently, warping is equivalent to stretching the frequency axis. More physical interpretation about it will be given in section IVB. Warping processing is based on a priori information of the environment via the warping function $w$. This warping function also depends on the mode $m$ that will be transformed into a Dirac, and will now be noted $w_{m}$.

Let consider a waveguide of depth $D$ with sound speed profile $c(z)$ depending of the depth $z$. Let define $c_{1}$ as the 
meaning of $c(z)$ in the water column. The warping function $w_{m}(f)$ for the mode $m$ is defined by its inverse $w_{m}^{-1}(f)$ :

$$
w_{m}^{-1}(f)=\frac{2 \pi}{c_{1}} k_{r m}(f)
$$

It is noticeable that for any mode $i$, if $i=m$ then $k_{r i}\left(w_{m}(f)\right)=2 \pi \frac{r}{c_{1}} f$; but if $i \neq m$ then $k_{r i}\left(w_{m}(f)\right)$ cannot be simplified. Then, the warped version of the spectrum of the received signal is:

$$
\mathbf{W}_{w_{m}} Y(f)=D_{m}(f) e^{j 2 \pi \frac{r}{c_{1}} f}+\sum_{i \neq m} D_{i}(f) e^{j r k_{r i}\left(w_{m}(f)\right)}
$$

where $D_{m}$ is a real functions giving the spectrum amplitude of the warped mode $m$. For the mode $m$, the phase of its spectrum $2 \pi \frac{r}{c_{1}} f$ is linear in frequency. It means that this mode is a Dirac in time. All its energy is concentrated on the time $r / c_{1}$ which corresponds to the direct path propagation time if the sound speed is constant in the water. The other modes are also warped but they are still spread in time (and thus different from a Dirac). This is shown in Figures 3 and 4. The first one shows the spectrogram of a received signal in an isovelocity waveguide (simulation). Figure 4 presents the spectrogram of the same signal, but after a frequency warping along the mode 3. It is well visible that after warping, mode 3 is a Dirac and its energy is concentrated around $t=1.2 \mathrm{~s}$.
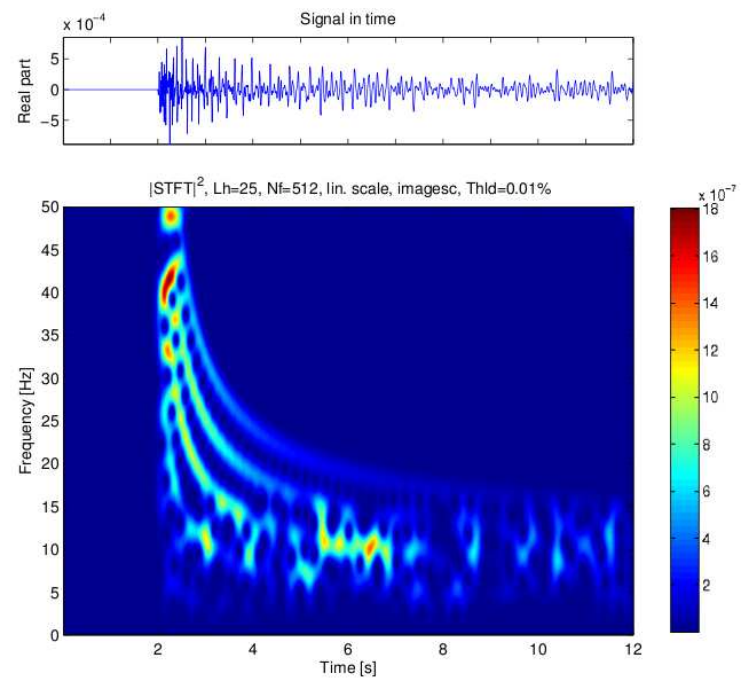

Fig. 3. Spectrogram of the received signal in an isovelocity waveguide

As frequency warping operates in the frequency domain, it does not required precise time-detection of the signal. It can be applied on any long signal containing the modal arrivals. If the source emits several pulses, it is possible to apply frequency warping only once on the received signal. All the arrivals will be warped at the same time.

\section{B. Physical interpretation}

In the received signal, modes are non-linear frequency modulations. Frequency warping processing transforms a given
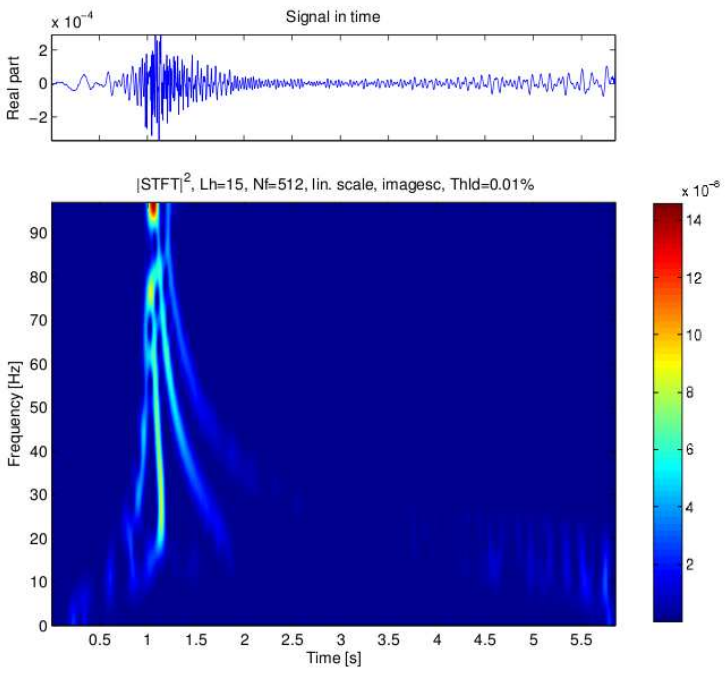

Fig. 4. Spectrogram of the warped signal (frequency warping along mode 3) in an isovelocity waveguide

mode into a Dirac (in time) by stretching frequency axis. In our configuration, the source emits a Dirac which is distorted by the dispersive propagation, and frequency warping transforms a given mode into a Dirac again. Consequently, frequency warping processing undoes the dispersion for a given mode. It is a post-reception treatment, but it can be though of as a transformation of the source. As the frequency dimension is stretched, the "equivalent-source" does not emit a pulse anymore but a frequency modulated signal which instantaneous frequency depends on the environment and on the mode number. Then for a given mode, the frequency law of the source is perfectly compensated by dispersive propagation. An experiment showing that is presented in section IV-C.

\section{Experimental tests on frequency warping processing}

1) Experimental protocol: Experiments about frequency warping processing are realized in an ultrasonic tank. It allows to do small scale experiment at high frequency in a perfectly controlled environment. Indeed, if distances (source/receiver distance and depth) are reduced by a factor $N$, frequency has to be multiplied by the same factor $N$ (the wavelength is then divided by $N$ ) so that the scale remains the same. The waveguide in the tank can be considered as a Pekeris one and its parameters are:

- Water column: sound speed $c_{1}=1487 \mathrm{~m} . \mathrm{s}^{-1}$, density $\rho_{1}=1000 \mathrm{~kg} \cdot \mathrm{m}^{-3}$, depth $D=1 \mathrm{~cm}$

- Bottom (stainless steel): sound speed $c_{2}=5800 \mathrm{~m} \cdot \mathrm{s}^{-1}$, density $\rho_{2}=7900 \mathrm{~kg} \cdot \mathrm{m}^{-3}$

To reproduce a classical sea configuration, the source is near below the surface and the receiver lay on the bottom. They are separated by a distance $r=90 \mathrm{~cm}$. The source emits a pulse of central frequency $1.5 \mathrm{MHz}$ and of bandwidth $3 \mathrm{MHz}$. Sampling frequency in reception is $10 \mathrm{MHz}$. This experimental protocol corresponds to a classical low frequency experiment in shallow water with a scale factor $N=10000$. 
2) Experimental results: The spectrogram of the received signal is presented in Figure 5. As modes overlap in time, they can barely be distinguished. Frequency warping processing is applied along mode 2, and spectrogram of the warped signal is presented in Figure 6. Mode 2 is a vertical line in the timefrequency plan located around time $t=3.5 \mathrm{~s}$, and thus it is a Dirac in time. Mode 1 which is low excited is on the left of mode 2 and the other modes are mixed on the right. To illustrate the physical interpretation given in section IV$\mathrm{B}$, the signal emited by the source is replaced by a chirp which frequency law will be compensated by dispersion for mode 2. The result is presented in Figure 7 and is equivalent as the one presented in Figure 6. This confirms the analogy between frequency warping (a post reception processing) and a modification of the source. This source modification creates a focus in time (here $t=3.5 \mathrm{~s}$ ) and can be seen as modal time reversal for a single receiver.

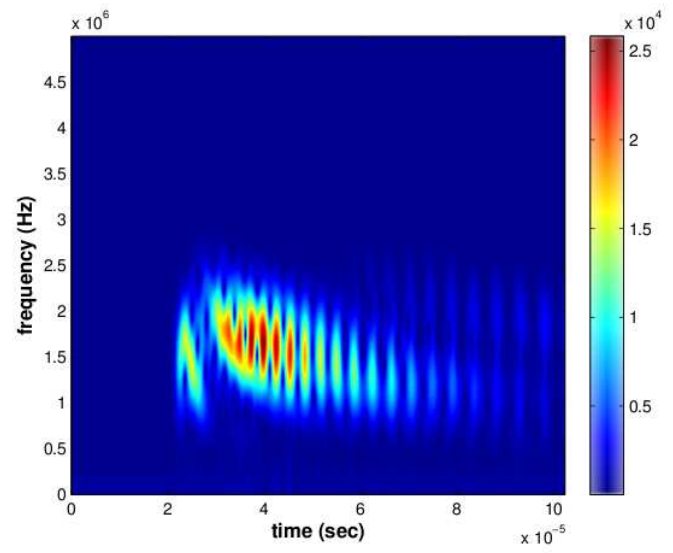

Fig. 5. Spectrogram of the received signal when the source emits a pulse (tank experiment)

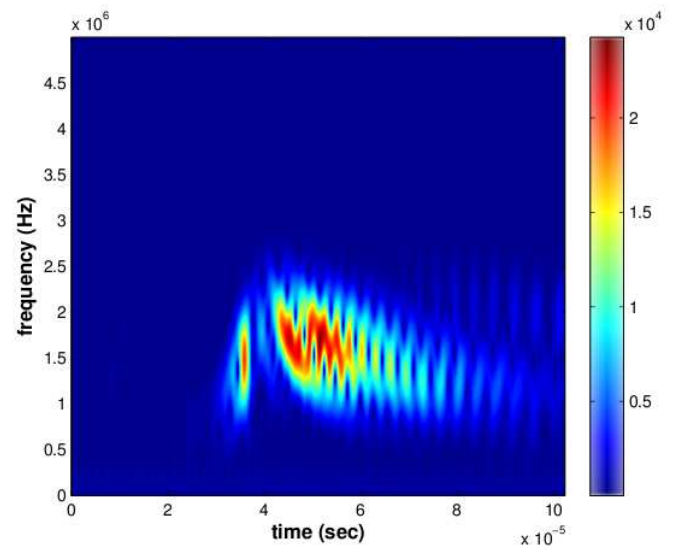

Fig. 6. Spectrogram of warped signal along mode 2 (tank experiment)

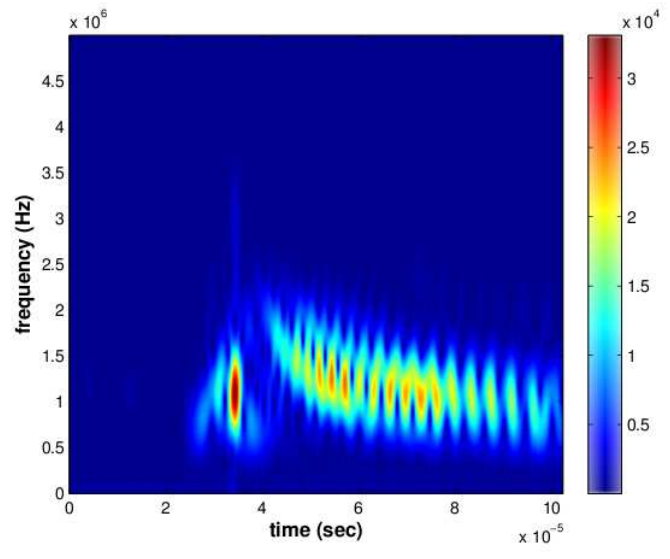

Fig. 7. Spectrogram of the received signal when the source emit a chirp (tank experiment)

\section{ENVIRONMENT CHARACTERIZATION}

\section{A. Principle}

Frequency warping transforms a given mode into a Dirac when the environment is well known. This transformation is really sensitive to environment mismatch: if there is an error on the a priori information of the environment, frequency warping is not accurate and the mode is not transformed into a real Dirac. This is an advantage when frequency warping is used to check if the a priori information of the environment is good. As a result, frequency warping can be used as a test in an inversion scheme on a single receiver:

1) Choose environmental parameter.

2) Apply frequency warping for every modes.

3) If the warped modes are not Diracs, go back to step 1.

In realistic case, an exhaustive search over all environment parameters is not possible. Consequently, such kind of algorithm requires optimization process to cleverly cover the parameters space. This is not presented in this article and is part of our future research. However, sections V-B will show that frequency warping is suitable for checking a priori information of the environment (step 2 of the algorithm).

\section{B. Example on simulated data}

To see how it is affected by environment mismatch, frequency warping processing is applied on simulated signal presented in section III which spectrogram is presented in Figure 2 . The simulated waveguide is a noisy Pekeris waveguide. The parameters of the simulation are:

- Water column: sound speed $c_{1}=1500 \mathrm{~m} \cdot \mathrm{s}^{-1}$, density $\rho_{1}=1000 \mathrm{~kg} \cdot \mathrm{m}^{-3}$, depth $D=130 \mathrm{~m}$

- Bottom: sound speed $c_{2}=2000 \mathrm{~m} \cdot \mathrm{s}^{-1}$, density $\rho_{2}=$ $2500 \mathrm{~kg} \cdot \mathrm{m}^{-3}$

- Depth of the source $z_{s}=20 \mathrm{~m}$

- Receiver on the bottom $z_{r}=130 \mathrm{~m}$

- Distance between source and receiver $r=4000 \mathrm{~m}$

- Sampling frequency $F_{s}=200 \mathrm{~Hz}$ 
- Signal to Noise Ratio $S N R=5 \mathrm{~dB}$ (gaussian white noise)

First, frequency warping is applied along mode 2 using the real environment parameters. The result is presented in Figure 8. Mode 2 is a perfectly vertical straight line on the timefrequency plan at time $0.5 \mathrm{~s}$; which means that as expected, mode 2 is a Dirac in time. Frequency warping processing has not been affected by the noise. Then, frequency warping is applied along mode 2, but a small error is introduced on the a priori information of the environment: the warping is applied with a water sound speed of $1515 \mathrm{~m} . \mathrm{s}^{-1}$ (an error of 5\%). The result is presented in Figure 9. This time, mode 2 is not perfectly vertical anymore and it lays around time $0.6 \mathrm{~s}$. Warping has been affected by the error on the a priori information. Same kind of result can be obtained if the error occurs on the bottom sound speed or the water depth. As a result, frequency warping sensitivity to environment mismatch can be used to characterize to waveguide.

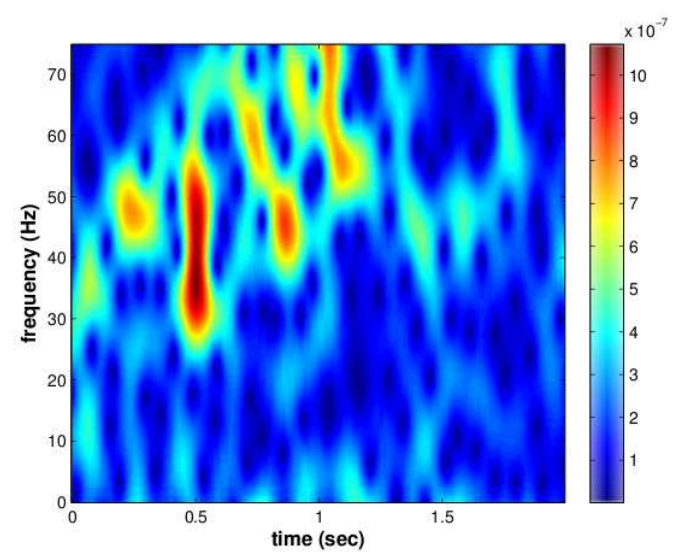

Fig. 8. Spectrogram of the simulated signal after warping without error on a priori information of the environment

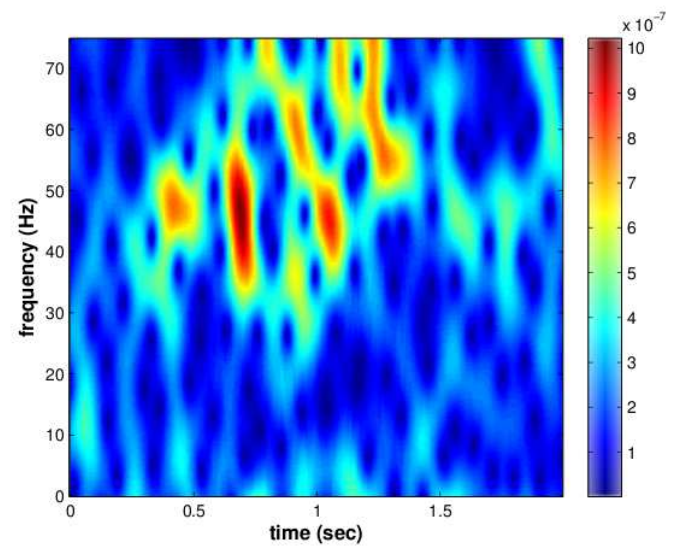

Fig. 9. Spectrogram of the simulated signal after warping with error on a priori information of the environment

\section{CONCLUSION}

Propagation of low frequency sound $(<200 \mathrm{~Hz})$ in shallow water $(<400 \mathrm{~m})$ is described by normal mode theory: the recorded pressure field can be decomposed into several modes. In case of impulsive sources (which can be natural as marine mammals or man-made as airgun), modes are non-linear frequency modulations and they share a common frequency band. For distance less than $20 \mathrm{~km}$, they are also overlapped in time. It makes it impossible to represent a received signal with classical tools such as time-frequency representation. This paper presents a new frequency warping processing which transforms a given mode into a Dirac, using a priori information of the environment. This transformation allows a better representation of the signal. Moreover, as frequency warping is sensitive to environment mismatch, it can be used as a tool to perform waveguide characterization.

\section{ACKNOWLEDGMENT}

The authors would like to thank W.A. Kuperman and S. Walker from Marine Physical Laboratory (Scripps Institution of Oceanography) for helpful discussions, DGA for founding this research, and Explora'Doc for founding displacements between GIPSA-Lab (Grenoble-INP, France) and Marine Physical Laboratory (Scripps Institution of Oceanography, USA).

\section{REFERENCES}

[1] C.O. Tiemann, M.B. Porter, and L.N. Frazer. Localization of marine mammals near Hawaii using an acoustic propagation model. The Journal of the Acoustical Society of America, 115:2834, 2004.

[2] J. Bonnel, G. Le Touzé, B. Nicolas, J.I. Mars, C. Gervaise. Automatic and passive whale localization in shallow water using gunshots. Oceans'08 MTS/IEEE, Quebec city, 2008.

[3] E.K. Skarsoulis, M.A. Kalogerakis. Ray-theoretic localization of an impulsive source in a stratified ocean using two hydrophones. The Journal of the Acoustical Society of America, 118:2934, 2005.

[4] J.P. Hermand. Broad-band geoacoustic inversion in shallow water from waveguideimpulse response measurements on a single hydrophone: theory and experimental results. IEEE Journal of Oceanic engineering, 24(1):41-66, 1999.

[5] S.M. Jesus, M.B. Porter, Y. Stephan, X. Demoulin, O.C. Rodriguez, E Coelho. Single hydrophone source localization. IEEE Journal of Oceanic Engineering, 25(3):337-346, 2000.

[6] J.I. Mars, J.L. Lacoume, G. Le Touzé, B. Nicolas. Matched representations and filters for guided waves. IEEE Journal of Oceanic Engineering, to be published.

[7] J. Bonnel, B. Nicolas, J.I. Mars, S.C. Walker. Estimation of modal arrival times with a single receiver for geoacoustic inversion in shallow water. IEEE Journal of Oceanic engineering, to be submited.

[8] F.B. Jensen. Computational ocean acoustics. Amer Inst of Physics, 1994.

[9] J.C. Hong, K.H. Sun, Y.Y. Kim. Dispersion-based short-time Fourier transform applied to dispersive wave analysis. The Journal of the Acoustical Society of America, 117:2949, 2005.

[10] R.G. Baraniuk, D.L. Jones. Unitary equivalence: A new twist on signal processing. IEEE transactions on signal processing, 43(10):2269-2282, 1995. 